\title{
$n$-step mingling inequalities: new facets for the mixed-integer knapsack set
}

\author{
Alper Atamtürk • Kiavash Kianfar
}

Received: 17 November 2009 / Accepted: 3 May 2010 / Published online: 25 May 2010

(C) The Author(s) 2010. This article is published with open access at Springerlink.com

\begin{abstract}
The $n$-step mixed integer rounding (MIR) inequalities of Kianfar and Fathi (Math Program 120(2):313-346, 2009) are valid inequalities for the mixedinteger knapsack set that are derived by using periodic $n$-step MIR functions and define facets for group problems. The mingling and 2-step mingling inequalities of Atamtürk and Günlük (Math Program 123(2):315-338, 2010) are also derived based on MIR and they incorporate upper bounds on the integer variables. It has been shown that these inequalities are facet-defining for the mixed integer knapsack set under certain conditions and generalize several well-known valid inequalities. In this paper, we introduce new classes of valid inequalities for the mixed-integer knapsack set with bounded integer variables, which we call $n$-step mingling inequalities (for positive integer $n$ ). These inequalities incorporate upper bounds on integer variables into $n$ step MIR and, therefore, unify the concepts of $n$-step MIR and mingling in a single class of inequalities. Furthermore, we show that for each $n$, the $n$-step mingling inequality defines a facet for the mixed integer knapsack set under certain conditions. For $n=2$, we extend the results of Atamtürk and Günlük on facet-defining properties of 2-step mingling inequalities. For $n \geq 3$, we present new facets for the mixed integer knapsack set. As a special case we also derive conditions under which the $n$-step MIR inequalities define facets for the mixed integer knapsack set. In addition, we show that
\end{abstract}

\footnotetext{
A. Atamtürk

Department of Industrial Engineering and Operations Research, University of California, Berkeley, CA 94720-1777, USA

e-mail: atamturk@berkeley.edu

K. Kianfar $(\varangle)$

Department of Industrial and Systems Engineering,

Texas A\&M University, College Station, TX 77843-3131, USA

e-mail:kianfar@tamu.edu
} 
$n$-step mingling can be used to generate new valid inequalities and facets based on covers and packs defined for mixed integer knapsack sets.

Keywords Mixed integer rounding · Mixed integer programming · Mingling · Valid inequality . Facet

\section{Mathematics Subject Classification (2000) 90C11}

\section{Introduction}

Mixed-integer rounding (MIR) is a simple yet powerful procedure for generating valid inequalities for mixed-integer programs (MIP) [14-16]. When used as cuts MIR inequalities are very effective for solving MIP with unbounded integer variables. However, for problems with bounded variables lifting techniques tend to be more effective as they explicitly use the variable bound information, whereas the MIR procedure does not. In order to incorporate bound information on the integer variables into MIR Atamtürk and Günlük [4] introduce a simple procedure, called mingling. Mingling updates the coefficients of a base inequality after arranging terms suitably by using upper bounds of the variables. They also define 2-step mingling inequalities, which subsume MIR inequalities properly and the 2-step MIR of Dash and Günlük [5] under certain conditions. Mingling and 2-step mingling lead to strong valid inequalities for mixed-integer sets with bounded variables and to facets of mixed-integer knapsack sets derived earlier by superadditive lifting techniques. In particular, mingling inequalities subsume continuous cover and inequalities of Marchand and Wolsey [12] for the mixed 0-1 knapsack problems; 2-step mingling inequalities subsume continuous integer knapsack cover and pack inequalities of Atamtürk [1,3] for mixed integer knapsack problems.

In another direction, for a base mixed-integer constraint Kianfar and Fathi [10] introduce a different generalization of MIR called the $n$-step MIR inequalities. These inequalities are obtained by applying periodic $n$-step MIR functions [10] on the coefficients of a base inequality. Kianfar and Fathi $[10,11]$ show that they are facet-defining for the infinite and finite group problems [6-9] and also for certain single-constraint mixed integer polyhedra. $n$-step MIR inequalities generalize the 2-step MIR inequalities of Dash and Günlük [5].

In this paper, we unify the concepts of mingling and $n$-step MIR to define a single class of valid inequalities, which we call the $n$-step mingling inequalities, for the mixed-integer knapsack set. These new inequalities incorporate upper bounds on integer variables into $(n-1)$-step MIR with the mingling procedure. While the 2-step mingling inequality does not subsume the 2-step MIR inequality properly, the 3 -step mingling inequality does. In general, $n$-step mingling inequalities subsume $(n-1)$-step MIR inequalities.

It is important to note that although the $(n-1)$-step MIR function is used to describe the coefficients of an $n$-step mingling inequality, the $n$-step mingling inequality is different from inequality one obtains by simply applying the $(n-1)$-step MIR procedure 
on a mingling inequality. Indeed, as we show later in the paper, the $n$-step mingling inequality dominates the latter inequality.

We show that for each positive integer $n$, the $n$-step mingling inequality defines a facet for the mixed-integer knapsack set under certain conditions. In the case of $n=2$, our results extend the results of [4] on facet-defining properties of 2-step mingling. For $n \geq 3$, our results present new facets for the mixed integer knapsack set. We also derive conditions under which the $n$-step MIR inequalities of [10] define facets for the mixed-integer knapsack set as a special case. In addition, we show that $n$-step mingling can be used to generate new valid inequalities and facets based on covers and packs defined for a mixed-integer knapsack set.

We begin with a brief review of mingling in Sect. 2. In Sect. 3 we introduce the basic ideas of $n$-step mingling by presenting 3 -step mingling inequality. We then present the general case of the $n$-step mingling inequality. In Sect. 4 we show that $n$-step mingling inequalities define new facets for the mixed-integer knapsack set under certain conditions, and also derive sufficient conditions under which the $n$-step MIR inequalities are facet-defining for this set. We present symmetric $n$-step mingling in Sect. 5 and $n$-step mingling cover and pack inequalities in Sect. 6 . We conclude with a few final remarks in Sect. 7.

\section{Mingling inequalities: a brief review}

In this section we briefly review the mingling and 2-step mingling inequalities of Atamtürk and Günlük [4]. This review establishes the notation that will be used in the rest of the paper. First, recall that for the mixed-integer set defined as

$$
\sum_{i \in N} a_{i} x_{i}+s \geq b, \quad x \in \mathbb{Z}_{+}^{N}, s \in \mathbb{R}_{+}
$$

the MIR inequality [14-16] with parameter $\alpha>0$ (also known as the $\alpha$-MIR inequality) is

$$
\sum_{i \in N} \mu_{\alpha, b}\left(a_{i}\right) x_{i}+s \geq \mu_{\alpha, b}(b),
$$

where $\mu_{\alpha, b}$ is the MIR function

$$
\mu_{\alpha, b}(t):=b^{(1)}\lfloor t / \alpha\rfloor+\min \left\{b^{(1)}, t^{(1)}\right\}
$$

where for any $r \in \mathbb{R}, r^{(1)}$ is defined as

$$
r^{(1)}:=r-\alpha\lfloor r / \alpha\rfloor
$$

We note that the nonnegativity of $x_{i}, i \in N$, is necessary for the validity of the MIR inequality unless $a_{i}$ is an integer multiple of $\alpha$, i.e. $a_{i} / \alpha \in \mathbb{Z}$; see $[4,5,10,13,16]$.

Now consider the mixed-integer knapsack set with a single continuous variable and upper bounds on the integer variables 


$$
K_{\geq}:=\left\{(x, s) \in \mathbb{Z}_{+}^{|N|} \times \mathbb{R}_{+}: \sum_{i \in I} a_{i} x_{i}+\sum_{j \in J} a_{j} x_{j}+s \geq b, x \leq u\right\}
$$

where $(I, J)$ is a partitioning of $N$ with $a_{i}>0$ for $i \in I a_{j}<0$ for $j \in J$. The upper bound on each integer variable can be either a positive integer or infinity. We assume in this section that $b \geq 0$. In Sect. 5 we will consider the case $b \leq 0$. It is clear that the MIR inequality (1) for $K_{\geq}$does not utilize the upper bounds $u$.

The mingling inequality for $K_{\geq}$does use the upper bounds $u$, and is derived as follows. Let $I^{+}=:\left\{1, \ldots, n^{+}\right\}$be a subset of $\left\{i \in I: a_{i}>b\right\}$ indexed in non-increasing order of $a_{i}$ 's, and

$$
\bar{J}:=\left\{j \in J: a_{j}+\sum_{i \in I^{+}} a_{i} u_{i}<0\right\}
$$

For $j \in J \backslash \bar{J}$, we define a set $I_{j}$, an integer $k_{j}$, and the numbers $\bar{u}_{i j}$ such that $0 \leq$ $\bar{u}_{i j} \leq u_{i}$ for $i \in I_{j}$ as follows:

$$
\begin{aligned}
I_{j} & :=\{1, \ldots, p(j)\}, \text { where } p(j):=\min \left\{p \in I^{+}: a_{j}+\sum_{i=1}^{p} a_{i} u_{i} \geq 0\right\} ; \\
k_{j} & :=\min \left\{k \in \mathbb{Z}_{+}: a_{j}+\sum_{i=1}^{p(j)-1} a_{i} u_{i}+a_{p(j)} k \geq 0\right\} ; \text { and } \\
\bar{u}_{i j} & := \begin{cases}u_{i}, & \text { if } i<p(j), \\
k_{j}, & \text { if } i=p(j) .\end{cases}
\end{aligned}
$$

On the other hand for $j \in \bar{J}$, we simply let $I_{j}:=I^{+}, p(j):=n^{+}, k_{j}:=u_{n^{+}}$, and $\bar{u}_{i j}:=u_{i}$ for $i \in I_{j}$. Also we define

$$
\delta_{j}:=\min \left\{b, a_{j}+\sum_{i \in I_{j}} a_{i} \bar{u}_{i j}\right\} \quad \text { for } j \in J
$$

and, therefore, we have $0 \leq \delta_{j} \leq b$ for $j \in J \backslash \bar{J}$, and $\delta_{j}<0$ for $j \in \bar{J}$. Also for $i \in I$, let $J_{i}:=\left\{j \in J: i \in I_{j}\right\}$; therefore, $J_{i}=\emptyset$ for $i \in I \backslash I^{+}$. Atamtürk and Günlük [4] prove that the mingling inequality

$$
\sum_{i \in I^{+}} b\left[x_{i}-\sum_{j \in J_{i}} \bar{u}_{i j} x_{j}\right]+\sum_{i \in I \backslash I^{+}} a_{i} x_{i}+\sum_{j \in J} \delta_{j} x_{j}+s \geq b
$$


is valid for $K_{\geq}$and facet-defining for $\operatorname{conv}\left(K_{\geq}\right)$provided that $b-\min \left\{\delta_{j}: j \in \bar{J}\right\} \geq$ $\max \left\{a_{i}: a_{i}>b, i \in I \backslash I^{+}\right\}$. For $K_{\geq}$they also introduce the 2-step mingling inequality

$$
\sum_{i \in I^{+}} \mu_{\alpha, b}(b)\left[x_{i}-\sum_{j \in J_{i}} \bar{u}_{i j} x_{j}\right]+\sum_{i \in I^{+} I^{+}} \mu_{\alpha, b}\left(a_{i}\right) x_{i}+\sum_{j \in J} \mu_{\alpha, b}\left(\delta_{j}\right) x_{j}+s \geq \mu_{\alpha, b}(b),
$$

which is valid for any $\alpha>0$ such that $\alpha\lceil b / \alpha\rceil \leq \alpha_{0}:=\min \left\{a_{i}: i \in I^{+}\right\}$. They show that if $b>0, \bar{J}=\emptyset, I^{+}=\left\{i \in I: a_{i} \geq \alpha\lceil b / \alpha\rceil\right\}$, and $\alpha=a_{k}$ for some $k \in I$, then inequality (3) becomes the continuous integer cover inequality [1] obtained by superadditive lifting [2], which has been shown to be facet-defining for $\operatorname{conv}\left(K_{\geq}\right)$in Atamtürk [1].

Observe that for $I^{+}=\varnothing$, the two-step mingling inequality (3) reduces to the $\alpha$-MIR inequality (1); and if $\bar{J}=\varnothing$ and $\alpha=\alpha_{0}$, then the two-step mingling inequality (3) reduces to the mingling inequality (2). Note that since $\left[x_{i}-\sum_{j \in J_{i}} \bar{u}_{i j} x_{j}\right]$ is not necessarily nonnegative, the 2-step mingling inequality (3) cannot be obtained by simply applying the MIR function $\mu_{\alpha, b}$ on the mingling inequality (2) considering $\left[x_{i}-\sum_{j \in J_{i}} \bar{u}_{i j} x_{j}\right]$ as a single integer variable.

\section{$3 n$-step mingling inequalities}

In this section we introduce the $n$-step mingling inequalities. inequalities. In order to present the basic ideas on an easier prove the validity of the 3-step mingling based on 2 -step mingling. After we establish this base case, we use induction on $n$ to prove the validity of $n$-step mingling for $n \geq 3$. Let us define some new notation that will be used throughout the paper. Let $\alpha=\left\{\alpha_{1}, \alpha_{2}, \ldots\right\}$ be a fixed sequence in $\mathbb{R}_{>0}$. Then for $r \in \mathbb{R}$ we define the following recursive remainders with respect to $\alpha$

$$
r^{(q)}:=r^{(q-1)}-\alpha_{q}\left\lfloor r^{(q-1)} / \alpha_{q}\right\rfloor
$$

where $r^{(0)}=r$. Based on the definition above, for any integer $q \geq 1$ and $b \in \mathbb{R}_{+}$we can define a partitioning of $\mathbb{R}$ as follows: for $m=0, \ldots, q-1$

$$
\begin{aligned}
& \mathcal{I}_{m}^{q}:=\left\{t \in \mathbb{R}: t^{(k)}<b^{(k)}, k=1, \ldots, m, t^{(m+1)} \geq b^{(m+1)}\right\} \\
& \mathcal{I}_{q}^{q}:=\left\{t \in \mathbb{R}: t^{(k)}<b^{(k)}, k=1, \ldots, q\right\} .
\end{aligned}
$$

For instance, for $q=1$

$$
t \in \begin{cases}\mathcal{I}_{0}^{1} & \text { if } t^{(1)} \geq b^{(1)} \\ \mathcal{I}_{1}^{1} & \text { if } t^{(1)}<b^{(1)}\end{cases}
$$


and for $q=2$

$$
t \in \begin{cases}\mathcal{I}_{0}^{2} & \text { if } t^{(1)} \geq b^{(1)} \\ \mathcal{I}_{1}^{2} & \text { if } t^{(1)}<b^{(1)} \text { and } t^{(2)} \geq b^{(2)} \\ \mathcal{I}_{2}^{2} & \text { if } t^{(1)}<b^{(1)} \text { and } t^{(2)}<b^{(2)}\end{cases}
$$

\subsection{3-step mingling}

Following the notation in Atamtürk and Günlük [4], for $j \in J$ define $\tilde{u}_{i j}:=\bar{u}_{i j}-1$ if $\delta_{j}=b$ and $i=p(j)$; and $\tilde{u}_{i j}:=\bar{u}_{i j}$ otherwise. We also define

$$
\tilde{\delta}_{j}:= \begin{cases}0, & \text { if } \delta_{j}=b \\ \delta_{j}, & \text { otherwise }\end{cases}
$$

In the process of proving validity of the 2-step mingling inequality in [4], it is proved that the inequality

$$
\sum_{i \in I^{+}} \alpha_{1}\left\lceil\frac{b}{\alpha_{1}}\right\rceil\left[x_{i}-\sum_{j \in J_{i}} \tilde{u}_{i j} x_{j}\right]+\sum_{i \in I \backslash I^{+}} a_{i} x_{i}+\sum_{j \in J} \tilde{\delta}_{j} x_{j}+s \geq b
$$

is valid for the set $K_{\geq}$if $\alpha_{1}\left\lceil b / \alpha_{1}\right\rceil \leq \alpha_{0}:=\min \left\{a_{i}: i \in I^{+}\right\}$. Inequality (4) can be relaxed to

$$
\begin{gathered}
-\alpha_{1}\left\lfloor\frac{b}{\alpha_{1}}\right\rfloor+\sum_{i \in I^{+}} \alpha_{1}\left\lceil\frac{b}{\alpha_{1}}\right\rceil\left[x_{i}-\sum_{j \in J_{i}} \tilde{u}_{i j} x_{j}\right]+\sum_{\substack{i \in I \backslash I^{+} \\
a_{i} \in \mathcal{I}_{0}^{1}}} \alpha_{1}\left\lceil\frac{a_{i}}{\alpha_{1}}\right\rceil x_{i}+\sum_{\substack{i \in I \backslash I^{+} \\
a_{i} \in \mathcal{I}_{1}^{1}}} \alpha_{1}\left\lfloor\frac{a_{i}}{\alpha_{1}}\right\rfloor x_{i} \\
+\sum_{\substack{i \in I \backslash I^{+} \\
a_{i} \in \mathcal{I}_{1}^{1}}} a_{i}^{(1)} x_{i}+\sum_{\substack{j \in J \\
\tilde{\delta}_{j} \in \mathcal{I}_{0}^{1}}} \alpha_{1}\left\lceil\frac{\tilde{\delta}_{j}}{\alpha_{1}}\right\rceil x_{j}+\sum_{\substack{j \in J \\
\tilde{\delta}_{j} \in \mathcal{I}_{1}^{1}}} \alpha_{1}\left[\frac{\tilde{\delta}_{j}}{\alpha_{1}}\right\rfloor x_{j}+\sum_{\substack{j \in J \\
\tilde{\delta}_{j} \in \mathcal{I}_{1}^{1}}} \tilde{\delta}_{j}^{(1)} x_{j}+s \geq b^{(1)},
\end{gathered}
$$

which can also be written as follows because when $\delta_{j}=b$ we have $\tilde{\delta}_{j}=0$ and $\tilde{u}_{i j}=\bar{u}_{i j}-1$.

$$
\begin{gathered}
\left.\left.-\alpha_{1}\left\lfloor\frac{b}{\alpha_{1}}\right\rfloor+\sum_{i \in I^{+}} \alpha_{1}\left\lceil\frac{b}{\alpha_{1}}\right\rceil\left[x_{i}-\sum_{j \in J_{i}} \bar{u}_{i j} x_{j}\right]+\sum_{\substack{i \in I \backslash I^{+} \\
a_{i} \in \mathcal{I}_{0}^{1}}} \alpha_{1}\left\lceil\frac{a_{i}}{\alpha_{1}}\right\rceil x_{i}+\sum_{\substack{i \in I \backslash I^{+} \\
a_{i} \in \mathcal{I}_{1}^{1}}} \alpha_{1}\right\rfloor \frac{a_{i}}{\alpha_{1}}\right\rfloor x_{i} \\
+\sum_{\substack{i \in I \backslash I^{+} \\
a_{i} \in \mathcal{I}_{1}^{1}}} a_{i}^{(1)} x_{i}+\sum_{\substack{j \in J \\
\delta_{j} \in \mathcal{I}_{0}^{1}}} \alpha_{1}\left\lceil\frac{\delta_{j}}{\alpha_{1}}\right] x_{j}+\sum_{\substack{j \in J \\
\delta_{j} \in \mathcal{I}_{1}^{1}}} \alpha_{1}\left\lfloor\frac{\delta_{j}}{\alpha_{1}}\right\rfloor x_{j}+\sum_{\substack{j \in J \\
\delta_{j} \in \mathcal{I}_{1}^{1}}} \delta_{j}^{(1)} x_{j}+s \geq b^{(1)} .
\end{gathered}
$$


On the other hand, the 2-step mingling inequality (3) in its open form can be written as

$$
\begin{aligned}
& -b^{(1)}\left\lfloor\frac{b}{\alpha_{1}}\right\rfloor+\sum_{i \in I^{+}} b^{(1)}\left\lceil\frac{b}{\alpha_{1}}\right\rceil\left[x_{i}-\sum_{j \in J_{i}} \bar{u}_{i j} x_{j}\right] \\
& +\sum_{\substack{i \in I \backslash I^{+} \\
a_{i} \in \mathcal{I}_{0}^{1}}} b^{(1)}\left\lceil\frac{a_{i}}{\alpha_{1}}\right\rceil x_{i}+\sum_{\substack{i \in I \backslash I^{+} \\
a_{i} \in \mathcal{I}_{1}^{1}}} b^{(1)}\left\lfloor\frac{a_{i}}{\alpha_{1}}\right\rfloor x_{i} \\
& +\sum_{\substack{i \in I \backslash I^{+} \\
a_{i} \in \mathcal{I}_{1}^{1}}} a_{i}^{(1)} x_{i}+\sum_{\substack{j \in J \\
\delta_{j} \in \mathcal{I}_{0}^{1}}} b^{(1)}\left\lceil\frac{\delta_{j}}{\alpha_{1}}\right] x_{j}+\sum_{\substack{j \in J \\
\delta_{j} \in \mathcal{I}_{1}^{1}}} b^{(1)}\left\lfloor\frac{\delta_{j}}{\alpha_{1}}\right\rfloor x_{j} \\
& +\sum_{\substack{j \in J \\
\delta_{j} \in \mathcal{I}_{1}^{1}}} \delta_{j}^{(1)} x_{j}+s \geq b^{(1)} .
\end{aligned}
$$

The last two inequalities imply that the following inequality is valid for any $b^{(1)} \leq$ $\gamma \leq \alpha_{1}$.

$$
\begin{gathered}
-\gamma\left\lfloor\frac{b}{\alpha_{1}}\right\rfloor+\sum_{i \in I^{+}} \gamma\left\lceil\frac{b}{\alpha_{1}}\right\rceil\left[x_{i}-\sum_{j \in J_{i}} \bar{u}_{i j} x_{j}\right]+\sum_{\substack{i \in I \backslash I^{+} \\
a_{i} \in \mathcal{I}_{0}^{1}}} \gamma\left\lceil\frac{a_{i}}{\alpha_{1}}\right\rceil x_{i}+\sum_{\substack{i \in I \backslash I^{+} \\
a_{i} \in \mathcal{I}_{1}^{1}}} \gamma\left\lfloor\frac{a_{i}}{\alpha_{1}}\right\rfloor x_{i} \\
+\sum_{\substack{i \in I \backslash I^{+} \\
a_{i} \in \mathcal{I}_{1}^{1}}} b^{(2)}\left\lceil\frac{a_{i}^{(1)}}{\alpha_{2}}\right\rceil x_{i}+\sum_{\substack{j \in J \\
\delta_{j} \in \mathcal{I}_{0}^{1}}} \gamma\left\lceil\frac{\delta_{j}}{\alpha_{1}}\right] x_{j}+\sum_{\substack{j \in J \\
\delta_{j} \in \mathcal{I}_{1}^{1}}} \gamma\left\lfloor\frac{\delta_{j}}{\alpha_{1}}\right\rfloor x_{j}+\sum_{\substack{j \in J \\
\delta_{j} \in \mathcal{I}_{1}^{1}}} \delta_{j}^{(1)} x_{j}+s \geq b^{(1)} .
\end{gathered}
$$

In particular, we can set $\gamma=\alpha_{2}\left\lceil\frac{b^{(1)}}{\alpha_{2}}\right\rceil$ as long as the condition $\alpha_{2}\left\lceil\frac{b^{(1)}}{\alpha_{2}}\right\rceil \leq \alpha_{1}$ is satisfied. Therefore, we will have

$$
\begin{aligned}
& -\alpha_{2}\left\lceil\frac{b^{(1)}}{\alpha_{2}}\right\rceil\left\lfloor\frac{b}{\alpha_{1}}\right\rfloor+\sum_{i \in I^{+}} \alpha_{2}\left\lceil\frac{b^{(1)}}{\alpha_{2}}\right\rceil\left\lceil\frac{b}{\alpha_{1}}\right\rceil\left[x_{i}-\sum_{j \in J_{i}} \bar{u}_{i j} x_{j}\right] \\
& +\sum_{\substack{i \in I \backslash I^{+} \\
a_{i} \in \mathcal{I}_{0}^{1}}} \alpha_{2}\left\lceil\frac{b^{(1)}}{\alpha_{2}}\right\rceil\left\lceil\frac{a_{i}}{\alpha_{1}}\right\rceil x_{i} \\
& \left.+\sum_{\substack{i \in I \backslash I^{+} \\
a_{i} \in \mathcal{I}_{1}^{1}}} \alpha_{2}\left\lceil\frac{b^{(1)}}{\alpha_{2}}\right\rceil\left\lfloor\frac{a_{i}}{\alpha_{1}}\right\rfloor x_{i}+\sum_{\substack{i \in I \backslash I^{+} \\
a_{i} \in \mathcal{I}_{1}^{1}}} a_{i}^{(1)} x_{i}+\sum_{j \in J} \alpha_{2}\left\lceil\frac{b^{(1)}}{\alpha_{2}}\right\rceil \mid \frac{\delta_{j}}{\alpha_{1}}\right\rceil \mathcal{I}_{0}^{1} \\
& \left.+\sum_{\substack{j \in J \\
\delta_{j} \in \mathcal{I}_{1}^{1}}} \alpha_{2}\left\lceil\frac{b^{(1)}}{\alpha_{2}}\right\rceil \mid \frac{\delta_{j}}{\alpha_{1}}\right\rfloor x_{j}+\sum_{\substack{j \in J \\
\delta_{j} \in \mathcal{I}_{1}^{1}}} \delta_{j}^{(1)} x_{j}+s \geq b^{(1)} .
\end{aligned}
$$


Now we apply the MIR function $\mu_{\alpha_{2}, b^{(1)}}$ to (7) considering $\left[x_{i}-\sum_{j \in J_{i}} \bar{u}_{i j} x_{j}\right]$ as a single integer variable. Note that this cannot be done in general since this expression is not necessarily nonnegative, however here since its coefficient is an integer multiple of the parameter $\alpha_{2}$, applying the MIR function gives a valid inequality as mentioned in Sect. 2. Doing so we arrive at the 3-step mingling inequality:

$$
\begin{aligned}
& \sum_{i \in I^{+}} b^{(2)}\left\lceil\frac{b^{(1)}}{\alpha_{2}}\right\rceil\left\lceil\frac{b}{\alpha_{1}}\right\rceil\left\lceil x_{i}-\sum_{j \in J_{i}} \bar{u}_{i j} x_{j}\right]+\sum_{\substack{i \in I \backslash I^{+} \\
a_{i} \in \mathcal{I}_{0}^{2}}} b^{(2)}\left\lceil\frac{b^{(1)}}{\alpha_{2}}\right\rceil\left\lceil\frac{a_{i}}{\alpha_{1}}\right\rceil x_{i} \\
& +\sum_{\substack{i \in I \backslash I^{+} \\
a_{i} \in \mathcal{I}_{1}^{2}}}\left(b^{(2)}\left\lceil\frac{b^{(1)}}{\alpha_{2}}\right\rceil\left\lfloor\frac{a_{i}}{\alpha_{1}}\right\rfloor+b^{(2)}\left\lceil\frac{a_{i}^{(1)}}{\alpha_{2}}\right\rceil\right) x_{i} \\
& +\sum_{\substack{i \in I \backslash I^{+} \\
a_{i} \in \mathcal{I}_{2}^{2}}}\left(b^{(2)}\left\lceil\frac{b^{(1)}}{\alpha_{2}}\right\rceil\left\lfloor\frac{a_{i}}{\alpha_{1}}\right\rfloor+b^{(2)}\left\lfloor\frac{a_{i}^{(1)}}{\alpha_{2}}\right\rfloor+a_{i}^{(2)}\right) x_{i} \\
& +\sum_{\substack{i \in J \\
\delta_{j} \in \mathcal{I}_{0}^{2}}} b^{(2)}\left\lceil\frac{b^{(1)}}{\alpha_{2}}\right\rceil\left\lceil\frac{\delta_{j}}{\alpha_{1}}\right\rceil x_{j}+\sum_{\substack{i \in J \\
\delta_{j} \in \mathcal{I}_{1}^{2}}}\left(b^{(2)}\left\lceil\frac{b^{(1)}}{\alpha_{2}}\right\rceil\left\lfloor\frac{\delta_{j}}{\alpha_{1}}\right\rfloor+b^{(2)}\left\lceil\frac{\delta_{j}^{(1)}}{\alpha_{2}}\right\rceil\right) x_{j} \\
& +\sum_{\substack{i \in J \\
\delta_{j} \in \mathcal{I}_{2}^{2}}}\left(b^{(2)}\left\lceil\frac{b^{(1)}}{\alpha_{2}}\right\rceil\left\lfloor\frac{\delta_{j}}{\alpha_{1}}\right\rfloor+b^{(2)}\left\lfloor\frac{\delta_{j}^{(1)}}{\alpha_{2}}\right\rfloor+\delta_{j}^{(2)}\right) x_{j}+s \geq b^{(2)}\left\lceil\frac{b^{(1)}}{\alpha_{2}}\right\rceil\left\lceil\frac{b}{\alpha_{1}}\right\rceil .
\end{aligned}
$$

For $\alpha=\left(\alpha_{1}, \alpha_{2}\right)$ and $b$, defining

$$
\mu_{\alpha, b}^{2}(t)= \begin{cases}\left\lceil\frac{b^{(1)}}{\alpha_{2}}\right\rceil\left\lceil\frac{t}{\alpha_{1}}\right\rceil b^{(2)} & \text { if } t \in \mathcal{I}_{0}^{2} \\ \left\lceil\frac{b^{(1)}}{\alpha_{2}}\right\rceil\left\lfloor\frac{t}{\alpha_{1}}\right\rfloor b^{(2)}+\left\lceil\frac{t^{(1)}}{\alpha_{2}}\right\rceil b^{(2)} & \text { if } t \in \mathcal{I}_{1}^{2} \\ \left\lceil\frac{b^{(1)}}{\alpha_{2}}\right\rceil\left\lfloor\frac{t}{\alpha_{1}}\right\rfloor b^{(2)}+\left\lfloor\frac{t^{(1)}}{\alpha_{2}}\right\rfloor b^{(2)}+t^{(2)} & \text { if } t \in \mathcal{I}_{2}^{2},\end{cases}
$$

the 3-step mingling inequality (8) can be written in a compact form as

$$
\begin{aligned}
& \sum_{i \in I^{+}} \mu_{\alpha, b}^{2}(b)\left[x_{i}-\sum_{j \in J_{i}} \bar{u}_{i j} x_{j}\right]+\sum_{i \in I \backslash I^{+}} \mu_{\alpha, b}^{2}\left(a_{i}\right) x_{i} \\
& \quad+\sum_{j \in J} \mu_{\alpha, b}^{2}\left(\delta_{j}\right) x_{j}+s \geq \mu_{\alpha, b}^{2}(b) .
\end{aligned}
$$


Hence, we have derived the 3-step mingling inequality, which is valid for $K_{\geq}$for $\alpha=\left(\alpha_{1}, \alpha_{2}\right)$ satisfying $\alpha_{i}\left\lceil b^{(i-1)} / \alpha_{i}\right\rceil \leq \alpha_{i-1}$ for $i=1,2$.

\section{$3.2 n$-step mingling}

The MIR function $\mu_{\alpha, b}$ and the 2-step MIR function $\mu_{\alpha, b}^{2}$ used above are special cases of the $n$-step MIR function for $n=1$ and $n=2$, respectively. The $n$-step MIR function was introduced in [10] as a tool for producing $n$-step MIR inequalities for a set that is slightly different from $K_{\geq}$, i.e., $Y=\left\{x \in \mathbb{Z}_{+}^{N}: \sum_{i=1}^{N} a_{i} x_{i}+\alpha_{1} z=b, z \in \mathbb{Z}\right\}$. With a small modification, for $K_{\geq}$we define the $n$-step MIR function for $\alpha=\left(\alpha_{1}, \ldots, \alpha_{n}\right)$ as follows:

$\mu_{\alpha, b}^{n}(t)= \begin{cases}\sum_{k=1}^{m} \prod_{l=k+1}^{n}\left\lceil\frac{b^{(l-1)}}{\alpha_{l}}\right\rceil\left\lfloor\frac{t^{(k-1)}}{\alpha_{k}}\right\rfloor b^{(n)}+\prod_{l=m+2}^{n}\left\lceil\frac{b^{(l-1)}}{\alpha_{l}}\right\rceil\left\lceil\frac{t^{(m)}}{\alpha_{m+1}}\right\rceil b^{(n)} & \text { if } t \in \mathcal{I}_{m}^{n} ; \\ \sum_{k=1}^{n} \prod_{l=k+1}^{n}\left\lceil\frac{b^{(l-1)}}{\alpha_{l}}\right\rceil\left\lfloor\frac{t^{(k-1)}}{\alpha_{k}}\right\rfloor b^{(n)}+t^{(n)} & \text { if } t \in 0,1, \ldots, n-1\end{cases}$

Accordingly, the $n$-step MIR inequality for $K_{\geq}$is

$$
\sum_{i \in N} \mu_{\alpha, b}^{n}\left(a_{i}\right) x_{i}+s \geq \mu_{\alpha, b}^{n}(b) .
$$

Clearly, inequality (10) does not use the the information on upper bounds $u$.

The argument for the validity of the 3-step mingling inequality can be generalized to prove the validity of what we will refer to as the n-step mingling inequality for $K_{\geq}$(Theorem 1 below). For $\alpha=\left(\alpha_{1}, \ldots, \alpha_{n-1}\right)$ satisfying $\alpha_{k}\left\lceil\frac{b^{(k-1)}}{\alpha_{k}}\right\rceil \leq \alpha_{k-1}$ for $k=1, \ldots, n-1$, the $n$-step mingling inequality is

$$
\begin{aligned}
& \sum_{i \in I^{+}} \mu_{\alpha, b}^{n-1}(b)\left[x_{i}-\sum_{j \in J_{i}} \bar{u}_{i j} x_{j}\right]+\sum_{i \in I \backslash I^{+}} \mu_{\alpha, b}^{n-1}\left(a_{i}\right) x_{i} \\
& +\sum_{j \in J} \mu_{\alpha, b}^{n-1}\left(\delta_{j}\right) x_{j}+s \geq \mu_{\alpha, b}^{n-1}(b) .
\end{aligned}
$$

We see that the $n$-step mingling inequality makes explicit use of the upper bound information. Observe that for $I^{+}=\emptyset, n$-step mingling inequality (11) reduces to the $(n-1)$-step MIR inequality (10). As in 2-step mingling, the $n$-step mingling inequality (for any $n$ ) cannot be obtained by simply applying $(n-1)$-step MIR function $\mu_{\alpha, b}^{n-1}$ on (2) by considering $\left[x_{i}-\sum_{j \in J_{i}} \bar{u}_{i j} x_{j}\right]$ as a single variable because this expression is not necessarily nonnegative.

Remark 1 Note that $(n-1)$-step MIR function is used to express the coefficients of the $n$-step mingling inequality. However, we should emphasize, that the $n$-step mingling inequality is different from inequality one obtains by simply applying the $(n-1)$ step MIR procedure on a mingling inequality. Indeed, the $n$-step mingling inequality dominates the latter as shown below. 
By collecting terms, let us rewrite the mingling inequality (2) in its unmingled form

$$
\sum_{i \in I^{+}} b x_{i}+\sum_{i \in I^{+} I^{+}} a_{i} x_{i}+\sum_{j \in J}\left(\delta_{j}-\sum_{i \in I_{j}} b \bar{u}_{i j}\right) x_{j}+s \geq b
$$

so that each term has a nonnegative variable. Then, the $(n-1)$-step MIR inequality for the mingling inequality is

$$
\sum_{i \in I^{+}} \mu_{\alpha, b}^{n-1}(b) x_{i}+\sum_{i \in I \backslash I^{+}} \mu_{\alpha, b}^{n-1}\left(a_{i}\right) x_{i}+\sum_{j \in J} \mu_{\alpha, b}^{n-1}\left(\delta_{j}-\sum_{i \in I_{j}} b \bar{u}_{i j}\right) x_{j}+s \geq \mu_{\alpha, b}^{n-1}(b) .
$$

Inequality (12) differs from the $n$-step mingling inequality (11) only in the coefficients of $x_{j}, j \in J$. Comparing these coefficients, by subadditivity of the $(n-1)$-step MIR function [10], we see that

$$
\begin{aligned}
\mu_{\alpha, b}^{n-1}\left(\delta_{j}\right) & \leq \mu_{\alpha, b}^{n-1}\left(\delta_{j}-\sum_{i \in I_{j}} b \bar{u}_{i j}\right)+\mu_{\alpha, b}^{n-1}\left(\sum_{i \in I_{j}} b \bar{u}_{i j}\right) \\
& \leq \mu_{\alpha, b}^{n-1}\left(\delta_{j}-\sum_{i \in I_{j}} b \bar{u}_{i j}\right)+\mu_{\alpha, b}^{n-1}(b) \sum_{i \in I_{j}} \bar{u}_{i j} .
\end{aligned}
$$

Therefore, the $(n-1)$-step MIR inequality for the mingling inequality is dominated by the $n$-step mingling inequality (11). Example 1 shows that the domination is strict.

Theorem 1 For $n \geq 2$, the $n$-step mingling inequality (11) is valid for $K_{\geq}$for $a$ sequence $\alpha_{k}\left\lceil\frac{b^{(k-1)}}{\alpha_{k}}\right\rceil \leq \alpha_{k-1}$ for $k=1, \ldots, n-1$.

Proof The case for $n=2$ was proved in [4]. For $n \geq 3$ consider the inequality

$$
\begin{aligned}
& -\alpha_{n-1} \prod_{l=1}^{n-1}\left\lceil\frac{b^{(l-1)}}{\alpha_{l}}\right\rceil+\alpha_{n-1}\left\lceil\frac{b^{(n-2)}}{\alpha_{n-1}}\right\rceil+\sum_{i \in I^{+}} \alpha_{n-1} \prod_{l=1}^{n-1}\left\lceil\frac{b^{(l-1)}}{\alpha_{l}}\right\rceil\left[x_{i}-\sum_{j \in J_{i}} \bar{u}_{i j} x_{j}\right] \\
& +\sum_{\substack{m=0 \\
a_{i} \in \mathcal{I}_{m}^{n-2}}}^{n-3} \sum_{\substack{+a^{+}}}\left(\sum_{k=1}^{m} \prod_{l=k+1}^{n-1}\left\lceil\frac{b^{(l-1)}}{\alpha_{l}}\right\rceil\left\lfloor\frac{a_{i}^{(k-1)}}{\alpha_{k}}\right\rfloor+\prod_{l=m+2}^{n-1}\left\lceil\frac{b^{(l-1)}}{\alpha_{l}}\right\rceil\left\lceil\frac{a_{i}^{(m)}}{\alpha_{m+1}}\right\rceil\right) x_{i} \\
& +\sum_{i \in I \backslash I^{+}}\left(\alpha_{n-1} \sum_{k=1}^{n-2} \prod_{l=k+1}^{n-1}\left\lceil\frac{b^{(l-1)}}{\alpha_{l}}\right\rceil\left\lfloor\frac{a_{i}{ }^{(k-1)}}{\alpha_{k}}\right\rfloor+a_{i}{ }^{(n-2)}\right) x_{i} \\
& a_{i} \in \mathcal{I}_{n-2}^{n-2}
\end{aligned}
$$




$$
\begin{aligned}
& +\sum_{m=0}^{n-3} \sum_{\substack{j \in J \\
\delta_{j} \in \mathcal{I}_{m}^{n-2}}} \alpha_{n-1}\left(\sum_{k=1}^{m} \prod_{l=k+1}^{n-1}\left\lceil\frac{b^{(l-1)}}{\alpha_{l}}\right\rceil\left\lfloor\frac{\delta_{j}^{(k-1)}}{\alpha_{k}}\right\rfloor+\prod_{l=m+2}^{n-1}\left\lceil\frac{b^{(l-1)}}{\alpha_{l}}\right\rceil\left\lceil\frac{\delta_{j}^{(m)}}{\alpha_{m+1}}\right\rceil\right) x_{j} \\
& \left.+\sum_{\substack{j \in J \\
\delta_{j} \in \mathcal{I}_{n-2}^{n-2}}}\left(\alpha_{n-1} \sum_{k=1}^{n-2} \prod_{l=k+1}^{n-1}\left\lceil\frac{b^{(l-1)}}{\alpha_{l}}\right\rceil \frac{\delta_{j}^{(k-1)}}{\alpha_{k}}\right\rfloor+\delta_{j}^{(n-2)}\right) x_{j}+s \geq b^{(n-2)}
\end{aligned}
$$

We have already shown in Sect. 3.1 that (13) and the $n$-step mingling inequality (11) are valid for $n=3$ (inequality (13) for $n=3$ reduces to inequality (7). Now we use induction on $n$. As the induction hypothesis we assume inequality (13) and the $n$-step mingling inequality are valid. We prove that inequality (13) is valid if $n$ is replaced with $n+1$ and then by applying a 1 -step MIR function we prove that the $(n+1)$-step mingling inequality is valid. Inequality (13) can be relaxed to the following inequality much like the way (4) is relaxed in Sect. 3.1:

$$
\begin{aligned}
& -\alpha_{n-1} \prod_{l=1}^{n-1}\left\lceil\frac{b^{(l-1)}}{\alpha_{l}}\right\rceil+\alpha_{n-1}+\sum_{i \in I^{+}} \alpha_{n-1} \prod_{l=1}^{n-1}\left\lceil\frac{b^{(l-1)}}{\alpha_{l}}\right\rceil\left[x_{i}-\sum_{j \in J_{i}} \bar{u}_{i j} x_{j}\right] \\
& +\sum_{m=0}^{n-2} \sum_{\substack{i \in I \backslash I^{+} \\
a_{i} \in \mathcal{I}_{m}^{n-1}}} \alpha_{n-1}\left(\sum_{k=1}^{m} \prod_{l=k+1}^{n-1}\left\lceil\frac{b^{(l-1)}}{\alpha_{l}}\right\rceil\left\lfloor\frac{a_{i}{ }^{(k-1)}}{\alpha_{k}}\right\rfloor+\prod_{l=m+2}^{n-1}\left\lceil\frac{b^{(l-1)}}{\alpha_{l}}\right\rceil\left\lceil\frac{a_{i}{ }^{(m)}}{\alpha_{m+1}}\right\rceil\right) x_{i} \\
& +\sum_{i \in I \backslash I^{+}}\left(\alpha_{n-1} \sum_{k=1}^{n-1} \prod_{l=k+1}^{n-1}\left\lceil\frac{b^{(l-1)}}{\alpha_{l}}\right\rceil\left\lfloor\frac{a_{i}{ }^{(k-1)}}{\alpha_{k}}\right\rfloor+a_{i}{ }^{(n-1)}\right) x_{i} \\
& a_{i} \in \mathcal{I}_{n-1}^{n-1} \\
& +\sum_{m=0}^{n-2} \sum_{\substack{j \in J \\
\delta_{j} \in \mathcal{I}_{m}^{n-1}}} \alpha_{n-1}\left(\sum_{k=1}^{m} \prod_{l=k+1}^{n-1}\left\lceil\frac{b^{(l-1)}}{\alpha_{l}}\right\rceil\left\lfloor\frac{\delta_{j}{ }^{(k-1)}}{\alpha_{k}}\right\rfloor+\prod_{l=m+2}^{n-1}\left\lceil\frac{b^{(l-1)}}{\alpha_{l}}\right\rceil\left\lceil\frac{\delta_{j}{ }^{(m)}}{\alpha_{m+1}}\right\rceil\right) x_{j} \\
& +\sum_{\substack{j \in J \\
\delta_{j} \in \mathcal{I}_{n-1}^{n-1}}}\left(\alpha_{n-1} \sum_{k=1}^{n-1} \prod_{l=k+1}^{n-1}\left\lceil\frac{b^{(l-1)}}{\alpha_{l}}\right\rceil\left\lfloor\frac{\delta_{j}{ }^{(k-1)}}{\alpha_{k}}\right\rfloor+\delta_{j}^{(n-1)}\right) x_{j}+s \geq b^{(n-1)} .
\end{aligned}
$$

On the other hand the $n$-step mingling inequality in its open form can be written as

$$
\begin{gathered}
-b^{(n-1)} \prod_{l=1}^{n-1}\left\lceil\frac{b^{(l-1)}}{\alpha_{l}}\right\rceil+b^{(n-1)}+\sum_{i \in I^{+}} b^{(n-1)} \prod_{l=1}^{n-1}\left\lceil\frac{b^{(l-1)}}{\alpha_{l}}\right\rceil\left\lceil x_{i}-\sum_{j \in J_{i}} \bar{u}_{i j} x_{j}\right] \\
\left.+\sum_{m=0}^{n-2} \sum_{\substack{i \in I \backslash I^{+} \\
a_{i} \in \mathcal{I}_{m}^{n-1}}} b^{(n-1)}\left(\sum_{k=1}^{m} \prod_{l=k+1}^{n-1}\left\lceil\frac{b^{(l-1)}}{\alpha_{l}}\right\rceil \mid \frac{a_{i}{ }^{(k-1)}}{\alpha_{k}}\right\rfloor+\prod_{l=m+2}^{n-1}\left\lceil\frac{b^{(l-1)}}{\alpha_{l}}\right\rceil\left\lceil\frac{a_{i}{ }^{(m)}}{\alpha_{m+1}}\right\rceil\right) x_{i}
\end{gathered}
$$




$$
\begin{aligned}
& +\sum_{\substack{i \in I \backslash I^{+} \\
a_{i} \in \mathcal{I}_{n-1}^{n-1}}}\left(b^{(n-1)} \sum_{k=1}^{n-1} \prod_{l=k+1}^{n-1}\left\lceil\frac{b^{(l-1)}}{\alpha_{l}}\right\rceil\left\lfloor\frac{a_{i}^{(k-1)}}{\alpha_{k}}\right\rfloor+a_{i}^{(n-1)}\right) x_{i} \\
& \left.\left.+\sum_{m=0}^{n-2} \sum_{\substack{j \in J \\
\delta_{j} \in \mathcal{I}_{m}^{n-1}}} b^{(n-1)}\left(\sum_{k=1}^{m} \prod_{l=k+1}^{n-1}\left\lceil\frac{b^{(l-1)}}{\alpha_{l}}\right\rceil \mid \frac{\delta_{j}^{(k-1)}}{\alpha_{k}}\right\rfloor+\prod_{l=m+2}^{n-1}\left\lceil\frac{b^{(l-1)}}{\alpha_{l}}\right\rceil \frac{\delta_{j}^{(m)}}{\alpha_{m+1}}\right\rceil\right) x_{j} \\
& \left.+\sum_{j \in J}\left(b^{(n-1)} \sum_{k=1}^{n-1} \prod_{l=k+1}^{n-1}\left\lceil\frac{b^{(l-1)}}{\alpha_{l}}\right\rceil \mid \frac{\delta_{j}(k-1)}{\alpha_{k}}\right\rfloor+\delta_{j}{ }^{(n-1)}\right) x_{j}+s \geq b^{(n-1)} .
\end{aligned}
$$

The only difference between (14) and (15) is that the multiplier $\alpha_{n-1}$ in front of the terms in (14) is replaced with $b^{(n-1)}$ in (15). Therefore the same valid inequality is valid if the multiplier $\alpha_{n-1}$ in (14) is replaced with any $\gamma$ satisfying $b^{(n-1)} \leq \gamma \leq \alpha_{n-1}$. In particular, since $b^{(n-1)} \leq \alpha_{n}\left\lceil\frac{b^{(n-1)}}{\alpha_{n}}\right\rceil \leq \alpha_{n-1}$ we can replace the multiplier $\alpha_{n-1}$ in (14) with $\alpha_{n}\left\lceil\frac{b^{(n-1)}}{\alpha_{n}}\right\rceil$ to arrive at the valid inequality

$$
\begin{aligned}
& -\alpha_{n} \prod_{l=1}^{n}\left\lceil\frac{b^{(l-1)}}{\alpha_{l}}\right\rceil+\alpha_{n}\left\lceil\frac{b^{(n-1)}}{\alpha_{n}}\right\rceil+\sum_{i \in I^{+}} \alpha_{n} \prod_{l=1}^{n}\left\lceil\frac{b^{(l-1)}}{\alpha_{l}}\right\rceil\left[x_{i}-\sum_{j \in J_{i}} \bar{u}_{i j} x_{j}\right]
\end{aligned}
$$

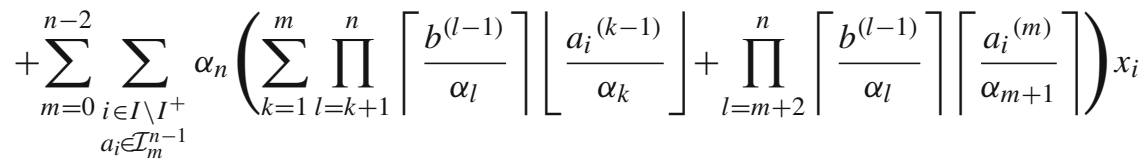

$$
\begin{aligned}
& +\sum_{i \in I \backslash I^{+}}\left(\alpha_{n} \sum_{k=1}^{n-1} \prod_{l=k+1}^{n}\left\lceil\frac{b^{(l-1)}}{\alpha_{l}}\right\rceil\left\lfloor\frac{a_{i}{ }^{(k-1)}}{\alpha_{k}}\right\rfloor+a_{i}{ }^{(n-1)}\right) x_{i} \\
& a_{i} \in \mathcal{I}_{n-1}^{n-1} \\
& +\sum_{m=0}^{n-2} \sum_{\substack{j \in J \\
\delta_{j} \in \mathcal{I}_{m}^{n-1}}} \alpha_{n}\left(\sum_{k=1}^{m} \prod_{l=k+1}^{n}\left\lceil\frac{b^{(l-1)}}{\alpha_{l}}\right\rceil\left\lceil\frac{\delta_{j}^{(k-1)}}{\alpha_{k}}\right\rfloor+\prod_{l=m+2}^{n}\left\lceil\frac{b^{(l-1)}}{\alpha_{l}}\right\rceil\left\lceil\frac{\delta_{j}{ }^{(m)}}{\alpha_{m+1}}\right\rceil\right) x_{j} \\
& +\sum_{\substack{j \in J \\
\delta_{j} \in \mathcal{I}_{n-1}^{n-1}}}\left(\alpha_{n} \sum_{k=1}^{n-1} \prod_{l=k+1}^{n}\left\lceil\frac{b^{(l-1)}}{\alpha_{l}}\right\rceil\left\lfloor\frac{\delta_{j}^{(k-1)}}{\alpha_{k}}\right\rfloor+\delta_{j}^{(n-1)}\right) x_{j}+s \geq b^{(n-1)} \text {. }
\end{aligned}
$$

We see that inequality (16) is the same as inequality (13) where $n$ is replaced with $n+1$. Now we apply $\mu_{\alpha_{n}, b^{(n-1)}}$ on inequality (16) considering $\left[x_{i}-\sum_{j \in J_{i}} \bar{u}_{i j} x_{j}\right]$ as a single integer variable. Although $\left[x_{i}-\sum_{j \in J_{i}} \bar{u}_{i j} x_{j}\right]$ is not necessarily nonnegative, application of $\mu_{\alpha_{n}, b^{(n-1)}}$ will give us a valid inequality because the coefficient of $\left[x_{i}-\sum_{j \in J_{i}} \bar{u}_{i j} x_{j}\right]$ is an integer multiple of $\alpha_{n}$. Doing so we get the $(n+1)$-step 
mingling inequality

$\sum_{i \in I^{+}} \mu_{\alpha, b}^{n}(b)\left[x_{i}-\sum_{j \in J_{i}} \bar{u}_{i j} x_{j}\right]+\sum_{i \in I \backslash I^{+}} \mu_{\alpha, b}^{n}\left(a_{i}\right) x_{i}+\sum_{j \in J} \mu_{\alpha, b}^{n}\left(\delta_{j}\right) x_{j}+s \geq \mu_{\alpha, b}^{n}(b)$,

which concludes the proof.

Remark 2 (A recursive formula for the $n$-step MIR function) If we generalize the notation introduced at the beginning of Sect. 3, we can write the $n$-step MIR function in a recursive form. Having the sequence of parameters $\left\{\alpha_{1}, \alpha_{2}, \ldots\right\}$, we can generalize the notation $r^{(q)}$ to $r^{\left(\alpha_{p}, \ldots, \alpha_{q}\right)}$ for $1 \leq p \leq q$ as follows

$$
r^{\left(\alpha_{p}, \ldots, \alpha_{q}\right)}:= \begin{cases}r^{\left(\alpha_{p}, \ldots, \alpha_{q-1}\right)}-\alpha_{q}\left\lfloor r^{\left(\alpha_{p}, \ldots, \alpha_{q-1}\right)} / \alpha_{q}\right\rfloor & \text { if } p \leq q \\ r-\alpha_{q}\left\lfloor r / \alpha_{q}\right\rfloor & \text { if } p=q\end{cases}
$$

Therefore $r^{(q)}=r^{\left(\alpha_{1}, \ldots, \alpha_{q}\right)}$. Using this notation it is easy to show that the $n$-step MIR function defined explicitly in Sect. 3.2 can be written recursively as follows

$$
\begin{aligned}
\mu_{\left(\alpha_{1}, \ldots, \alpha_{n}\right), b}^{n}(t)= & \mu_{\left(\alpha_{2}, \ldots, \alpha_{n}\right), b^{\left(\alpha_{1}\right)}}^{n-1}\left(b^{\left(\alpha_{1}\right)}\right)\left\lfloor\frac{t}{\alpha_{1}}\right\rfloor \\
& +\min \left\{\mu_{\left(\alpha_{2}, \ldots, \alpha_{n}\right), b^{\left(\alpha_{1}\right)}}^{n-1}\left(b^{\left(\alpha_{1}\right)}\right), \mu_{\left(\alpha_{2}, \ldots, \alpha_{n}\right), b^{\left(\alpha_{1}\right)}}^{n-1}\left(t^{\left(\alpha_{1}\right)}\right)\right\},
\end{aligned}
$$

where $\mu_{\alpha_{1}, b}^{1}(t)=b^{\left(\alpha_{1}\right)}\left\lfloor\frac{t}{\alpha_{1}}\right\rfloor+\min \left\{b^{\left(\alpha_{1}\right)}, t^{\left(\alpha_{1}\right)}\right\}$ as defined in (1).

\section{4 -step mingling facets for mixed-integer knapsack sets}

As our next main result, in this section we prove that, for any $n$, the $n$-step mingling inequalities are facet-defining for the mixed integer knapsack set under certain conditions. This makes $n$-step mingling a new way to generate facets for this set. Facets generated by $n$-step mingling for $n \geq 3$ (and also $n=2$ where $\bar{J} \neq \emptyset$ ) were not introduced in the literature before.

Theorem 2 For $n \geq 2$, the $n$-step mingling inequality (11) is facet-defining for $\operatorname{conv}\left(K_{\geq}\right)$if the following conditions are satisfied:

(i) $b^{(n-1)}>0$ and $\alpha_{k}=a_{i_{k}}$ where $i_{k} \in I \backslash I^{+}$for $k=1, \ldots, n-1$;

(ii) $I^{+}=\left\{i \in I: a_{i} \geq \alpha_{1}\left\lceil b / \alpha_{1}\right\rceil\right\}$ and $\alpha_{k-1} \geq \alpha_{k}\left\lceil\frac{b^{(k-1)}}{\alpha_{k}}\right\rceil$ for $k=2, \ldots, n-1$;

(iii) $u_{i_{1}} \geq\left\lceil\frac{b}{\alpha_{1}}\right\rceil-\left\lceil\frac{\min \left\{\delta_{j}: j \in \bar{J}\right\}}{\alpha_{1}}\right\rceil$ and $u_{i_{k}} \geq\left\lceil\frac{b^{(k-1)}}{\alpha_{k}}\right\rceil$ for $k=2, \ldots, n-1$.

Proof The validity of $n$-step mingling was proved in Theorem 1 . Regarding condition (i), define $I_{\alpha}$ as the subset of $I$ that its corresponding coefficients are the parameters $\alpha_{1}, \ldots, \alpha_{n-1}$, i.e. $I_{\alpha}:=\left\{i_{1}, \ldots, i_{n-1}\right\}$. Below we list $|I|+|J|+1$ affinely 
independent points in $K_{\geq}$on the face defined by (11). For each point we only describe the nonzero $x$ components.

- The point $P_{0}=(x, s)$ such that $s=b^{(n-1)} ; \quad x_{i_{k}}=\left\lfloor\frac{b^{(k-1)}}{\alpha_{k}}\right\rfloor$ for $k=1, \ldots, n-1 ;$

- For each $i_{m} \in I_{\alpha}$ where $m \in\{1, \ldots, n-1\}$, the point $P_{i}=(x, s)$ such that $s=0 ; \quad x_{i_{k}}=\left\lfloor\frac{b^{(k-1)}}{\alpha_{k}}\right\rfloor$ for $k=1, \ldots, m-1 ; \quad x_{i_{m}}=\left\lceil\frac{b^{(m-1)}}{\alpha_{m}}\right\rceil ;$

- For each $i \in I^{+}$, the point $P_{i}=(x, s)$ such that $s=0 ; \quad x_{i}=1$;

- For each $i \in I \backslash\left(I^{+} \cup I_{\alpha}\right)$ where $a_{i} \in \mathcal{I}_{m}^{n-1}$ and $m \in\{0, \ldots, n-2\}$, the point $P_{i}=(x, s)$ such that

$s=0 ; \quad x_{i}=1 ; \quad x_{i_{k}}=\left\lceil\frac{b^{(k-1)}}{\alpha_{k}}\right\rceil-\left\lceil\frac{a_{i}{ }^{(k-1)}}{\alpha_{k}}\right\rceil$ for $k=1, \ldots, m+1 ;$

- For each $i \in I \backslash\left(I^{+} \cup I_{\alpha}\right)$ where $a_{i} \in \mathcal{I}_{n-1}^{n-1}$, the point $P_{i}=(x, s)$ such that

$$
s=b^{(n-1)}-a_{i}{ }^{(n-1)} ; \quad x_{i}=1 ; \quad x_{i_{k}}=\left\lceil\frac{b^{(k-1)}}{\alpha_{k}}\right\rceil-\left\lceil\frac{a_{i}{ }^{(k-1)}}{\alpha_{k}}\right\rceil \text { for } k=1, \ldots, n-1 ;
$$

- For each $j \in J$ where $\delta_{j}<b$ and $\delta_{j} \in \mathcal{I}_{m}^{n-1}$ and $m \in\{0, \ldots, n-2\}$, the point $P_{j}=(x, s)$ such that

$$
\begin{aligned}
& s=0 ; \quad x_{j}=1 ; \quad x_{i}=\bar{u}_{i j} \text { for } i \in I_{j} ; \quad x_{i_{k}}=\left\lceil\frac{b^{(k-1)}}{\alpha_{k}}\right\rceil-\left\lceil\frac{\delta_{j}^{(k-1)}}{\alpha_{k}}\right\rceil \text { for } k= \\
& 1, \ldots, m+1 ;
\end{aligned}
$$

- For each $j \in J$ where $\delta_{j}<b$ and $\delta_{j} \in \mathcal{I}_{n-1}^{n-1}$, the point $P_{j}=(x, s)$ such that $s=b^{(n-1)}-\delta_{j}^{(n-1)} ; x_{j}=1 ; x_{i}=\bar{u}_{i j}$ for $i \in I_{j} ; x_{i_{k}}=\left\lceil\frac{b^{(k-1)}}{\alpha_{k}}\right\rceil-\left\lceil\frac{\delta_{j}^{(k-1)}}{\alpha_{k}}\right\rceil$ for $k=1, \ldots, n-1$;

- For each $j \in J$ where $\delta_{j}=b$, the point $P_{j}=(x, s)$ such that $s=0 ; \quad x_{j}=1 ; \quad x_{i}=\bar{u}_{i j}$ for $i \in I_{j}$;

It is not difficult to verify that given the conditions (i) to (iii) all these points belong to $\operatorname{conv}\left(K_{\geq}\right)$and satisfy (11) at equality. Furthermore these points are affinely independent because if we write them as rows of a square matrix, the rows and columns of the matrix can be rearranged to obtain a nonsingular lower triangular matrix. This can be done as follows: The columns of the matrix correspond to the variables in the vector $(x, s)$. We arrange these columns from left to right in the order $x_{i}: i \in I^{+}, x_{i}: i \in I_{\alpha}, s, x_{i} \in i \in I \backslash\left(I^{+} \cup I_{\alpha}\right)$, and $x_{j}: j \in J$, where indices within each of the sets $I^{+}, I_{\alpha}, I \backslash\left(I^{+} \cup I_{\alpha}\right)$, and $J$ are in increasing order. Now if we arrange the rows from top to bottom as $P_{i}: i \in I^{+}, P_{i}: i \in I_{\alpha}, P_{0}, P_{i} \in i \in I \backslash\left(I^{+} \cup I_{\alpha}\right)$, and $P_{j}: j \in J$, where the order of indices within each set is increasing, we arrive at a lower triangular matrix. Moreover, the elements on the diagonal are nonzero. This concludes the proof.

Note that condition (i) ensures that $\alpha_{k}<\alpha_{0}$ for $k=1, \ldots, n-1$. This is a natural choice, because as by condition (ii) we have $\alpha_{1} \geq \alpha_{2} \geq \cdots \geq \alpha_{n-1}$ and $I^{+}=$ $\left\{i \in I: a_{i} \geq \alpha_{1}\left\lceil b / \alpha_{1}\right\rceil\right\}$, if $i_{p} \in I^{+}$, we must have $i_{1}, \ldots, i_{p} \in I^{+}$and $b \leq a_{i_{1}}=$ $a_{i_{2}}=\cdots=a_{i_{p}}\left(\right.$ or $\left.b \leq \alpha_{0}=\alpha_{1}=\alpha_{2}=\cdots=\alpha_{p}\right)$. It is easy to verify that if this 
is true and $\bar{J}=\emptyset$, the $n$-step mingling inequality with $\alpha=\left(\alpha_{1}, \ldots, \alpha_{n-1}\right)$ reduces to $(n-p)$-step mingling inequality with $\alpha=\left(\alpha_{p+1}, \ldots, \alpha_{n-1}\right)$. However, if $\bar{J} \neq \emptyset$, inequalities are not comparable.

We also note that 2-step mingling inequalities are observed to be facet-defining in [4] when $\bar{J}=\varnothing$, in which case they are equivalent to the continuous cover inequalities for $K_{\geq}$, which have been shown to be facet-defining by superadditive lifting of a simple MIR inequality in [1]. Therefore, for the particular case of 2-step mingling, Theorem 2 extends the facet-defining property to the case where $\bar{J} \neq \emptyset$ with an alternative direct proof.

Below we present a numerical example illustrating the facets obtained by mingling, 2-step, and 3-step mingling and compare them with a direct application of 1-step and 2-step MIR on the mingling inequality. Higher step mingling inequalities can also be generated similarly.

Example 1 Consider the set $K_{\geq}$defined by the inequality

$$
37 x_{1}+33 x_{2}+31 x_{3}+15 x_{4}+13 x_{5}+6 x_{6}-63 x_{7}-82 x_{8}-107 x_{9}+s \geq 25
$$

and let $u$ be the vector of upper bounds and $u_{1}=u_{2}=u_{3}=1$. We have $I=$ $\{1,2,3,4,5,6\}$ and $J=\{7,8,9\}$. For $I^{+}=\left\{i \in I: a_{i}>b\right\}=\{1,2,3\}$, we have $\bar{J}=\{9\}, I_{7}=\{1,2\}, I_{8}=I_{9}=\{1,2,3\}$, and so $J_{1}=J_{2}=\{7,8,9\}$ and $J_{3}=$ $\{8,9\}$. Also $\delta_{7}=7, \delta_{8}=19, \delta_{9}=-6$. Then the corresponding mingling inequality (2) is

$$
25 x_{1}+25 x_{2}+25 x_{3}+15 x_{4}+13 x_{5}+6 x_{6}-43 x_{7}-56 x_{8}-81 x_{9}+s \geq 25,
$$

which is facet-defining based on the choice of $I^{+}$and Proposition 2 of [4]. In order to write a 2 -step mingling inequality, we maychoose $\alpha_{1}=a_{4}=15$. So that we have a facet-defining inequality, based on Theorem 2 , we choose $I^{+}=\left\{i \in I: a_{i} \geq \alpha_{1}\left\lceil b / \alpha_{1}\right\rceil\right\}=$ $\{1,2,3\}$. Then the sets $\bar{J}, I_{j}$, and $J_{i}$ and the values of $\delta_{j}$ 's are the same as above and conditions (i) and (ii) of Theorem 2 are satisfied. For condition (iii) to hold, we need $u_{4} \geq\left\lceil\frac{b}{\alpha_{1}}\right\rceil-\left\lceil\frac{\min \left\{\delta_{j}: j \in \bar{J}\right\}}{\alpha_{1}}\right\rceil=2$. In this case, $b, a_{5} \in \mathcal{I}_{0}^{1}$ and $a_{4}, a_{6}, \delta_{7}, \delta_{8}, \delta_{9} \in \mathcal{I}_{1}^{1}$. So the corresponding 2-step mingling inequality (3) is

$$
20 x_{1}+20 x_{2}+20 x_{3}+10 x_{4}+10 x_{5}+6 x_{6}-33 x_{7}-46 x_{8}-61 x_{9}+s \geq 20,
$$

which defines a facet if $u_{4} \geq 2$. Based on Theorem 2 the list of 10 affinely independent feasible points that lie on this facet is as follows. They are arranged in a form that shows the lower triangular structure, and hence affine independence. 


$$
\left[\begin{array}{llllllllll}
x_{1} & x_{2} & x_{3} & x_{4} & s & x_{5} & x_{6} & x_{7} & x_{8} & x_{9} \\
1 & 0 & 0 & 0 & 0 & 0 & 0 & 0 & 0 & 0 \\
0 & 1 & 0 & 0 & 0 & 0 & 0 & 0 & 0 & 0 \\
0 & 0 & 1 & 0 & 0 & 0 & 0 & 0 & 0 & 0 \\
0 & 0 & 0 & 2 & 0 & 0 & 0 & 0 & 0 & 0 \\
0 & 0 & 0 & 1 & 10 & 0 & 0 & 0 & 0 & 0 \\
0 & 0 & 0 & 1 & 0 & 1 & 0 & 0 & 0 & 0 \\
0 & 0 & 0 & 1 & 4 & 0 & 1 & 0 & 0 & 0 \\
1 & 1 & 0 & 1 & 3 & 0 & 0 & 1 & 0 & 0 \\
1 & 1 & 1 & 0 & 6 & 0 & 0 & 0 & 1 & 0 \\
1 & 1 & 1 & 2 & 1 & 0 & 0 & 0 & 0 & 1
\end{array}\right]
$$

On the other hand, the MIR inequality for the mingling inequality (17) is

$$
20 x_{1}+20 x_{2}+20 x_{3}+10 x_{4}+10 x_{5}+6 x_{6}-28 x_{7}-36 x_{8}-51 x_{9}+s \geq 20 \text {, }
$$

which is strictly dominated by the 2-step mingling inequality (18) as shown in Remark 1.

The 3-step mingling inequality can be constructed by choosing $\alpha_{1}=a_{4}=15$ and $\alpha_{2}=a_{6}=6$. For it to be facet-defining, the choice of $I^{+}$will be the same as above. All other sets will remain the same as well. In order to satisfy condition (iii) of Theorem 2, we need $u_{4} \geq 2$ and $u_{6} \geq\left\lceil\frac{b^{(1)}}{\alpha_{2}}\right\rceil=2$. In this case, $b \in \mathcal{I}_{0}^{2}$ and $a_{4}, a_{5}, a_{6}, \delta_{7}, \delta_{8}, \delta_{9} \in \mathcal{I}_{2}^{2}$. Then the corresponding 3-step mingling inequality (9) is

$$
16 x_{1}+16 x_{2}+16 x_{3}+8 x_{4}+8 x_{5}+4 x_{6}-27 x_{7}-36 x_{8}-49 x_{9}+s \geq 16,
$$

and it defines a facet if $u_{4}, u_{6} \geq 2$. The list of 10 affinely independent feasible points that lie on this facet according to Theorem 2 is as follows:

$$
\left[\begin{array}{llllllllll}
x_{1} & x_{2} & x_{3} & x_{4} & x_{6} & s & x_{5} & x_{7} & x_{8} & x_{9} \\
1 & 0 & 0 & 0 & 0 & 0 & 0 & 0 & 0 & 0 \\
0 & 1 & 0 & 0 & 0 & 0 & 0 & 0 & 0 & 0 \\
0 & 0 & 1 & 0 & 0 & 0 & 0 & 0 & 0 & 0 \\
0 & 0 & 0 & 2 & 0 & 0 & 0 & 0 & 0 & 0 \\
0 & 0 & 0 & 1 & 2 & 0 & 0 & 0 & 0 & 0 \\
0 & 0 & 0 & 1 & 1 & 4 & 0 & 0 & 0 & 0 \\
0 & 0 & 0 & 1 & 0 & 0 & 1 & 0 & 0 & 0 \\
1 & 1 & 0 & 1 & 0 & 3 & 0 & 1 & 0 & 0 \\
1 & 1 & 1 & 0 & 1 & 0 & 0 & 0 & 1 & 0 \\
1 & 1 & 1 & 2 & 0 & 1 & 0 & 0 & 0 & 1
\end{array}\right]
$$

On the other hand, the 2-step MIR inequality for the mingling inequality (17) is

$$
16 x_{1}+16 x_{2}+16 x_{3}+8 x_{4}+8 x_{5}+4 x_{6}-22 x_{7}-28 x_{8}-41 x_{9}+s \geq 16,
$$


which is strictly dominated by the 3-step mingling inequality (19) as shown in Remark 1.

Theorem 2 also makes it possible to derive sufficient facet-defining conditions for the $n$-step MIR inequalities of [10]. In the $(n+1)$-step mingling inequality, if we choose $I^{+}=\emptyset$, we obtain the $n$-step MIR inequality. Using this fact and based on Theorem 2, we can state the following result about facet-defining property of $n$-step MIR inequalities for $K_{\geq}$:

Corollary 1 The $n$-step MIR inequality (10) defines a facet for conv $\left(K_{\geq}\right)$if the following conditions are satisfied:

(i) $b^{(n)}>0, \alpha_{k}=a_{i_{k}}$ where $i_{k} \in I$ for $k=1, \ldots, n$;

(ii) $a_{i} \leq \alpha_{1}\left\lceil b / \alpha_{1}\right\rceil$ for all $i \in I$, and $\alpha_{k-1} \geq \alpha_{k}\left\lceil\frac{b^{(k-1)}}{\alpha_{k}}\right\rceil$ for $k=2, \ldots, n$;

(iii) $u_{i_{1}} \geq\left\lceil\frac{b}{\alpha_{1}}\right\rceil-\left\lceil\frac{\min \left\{a_{j}: j \in J\right\}}{\alpha_{1}}\right\rceil$ and $u_{i_{k}} \geq\left\lceil\frac{b^{(k-1)}}{\alpha_{k}}\right\rceil$ for $k=2, \ldots, n$.

\section{Symmetric $n$-step mingling inequalities}

In this section we give a symmetric form of the $n$-step mingling inequalities for $K_{\geq}$ for the case $b \leq 0$. The approach is similar to the one used in [4] and is based on the correspondence between valid inequalities and facets for $K_{\geq}$and

$$
K_{\leq}=\left\{(x, t) \in \mathbb{Z}_{+}^{N} \times \mathbb{R}_{+}: a x \leq b+t, x \leq u\right\}
$$

Lemma 1 [4] The inequality $\pi x+s \geq \pi_{0}$ is valid for $K_{\geq}$if and only if inequality $(a-\pi) x \leq b-\pi_{0}+t$ is valid for $K_{\leq}$. Moreover, $\pi x+s \geq \pi_{0}$ is facet-defining for $\operatorname{conv}\left(K_{\geq}\right)$if and only if $(a-\pi) x \leq b-\pi_{0}+t$ is facet-defining for $\operatorname{conv}\left(K_{\leq}\right)$.

To write the symmetric $n$-step mingling inequality we update the coefficients of $x_{i}, i \in I$, in the base inequality of $K_{\geq}$using the upper bounds of $x_{j}, j \in J$. Let $J^{-}=:\left\{1, \ldots, n^{-}\right\}$be a subset of $\left\{j \in \bar{J}: a_{j}<b\right\}$ indexed in nondecreasing order of $a_{j}$ 's, and $\bar{I}:=\left\{i \in I: a_{i}+\sum_{i \in J^{-}} a_{j} u_{j}>0\right\}$. For any $i \in I \backslash \bar{I}$, we define a set $J_{i}$, an integer $k_{i}$, and the numbers $0 \leq \bar{u}_{j i} \leq u_{j}$ for $j \in J_{i}$ as follows:

$$
\begin{aligned}
J_{i} & :=\{1, \ldots, p(i)\}, \text { where } p(i):=\min \left\{p \in J^{-}: a_{i}+\sum_{j=1}^{p} a_{j} u_{j} \leq 0\right\} ; \\
k_{i} & :=\min \left\{k \in \mathbb{Z}_{+}: a_{i}+\sum_{j=1}^{p(i)-1} a_{j} u_{j}+a_{p(i)} k \leq 0\right\} ; \text { and } \\
\bar{u}_{j i} & := \begin{cases}u_{i}, & \text { if } j<p(i), \\
k_{i}, & \text { if } j=p(i) .\end{cases}
\end{aligned}
$$

For $i \in \bar{I}$, we let $J_{i}:=J^{-}, p(i):=n^{-}, k_{i}:=u_{n^{-}}$, and $\bar{u}_{j i}:=u_{j}$ for $j \in J_{i}$. As a result, if we define 


$$
\lambda_{i}:=\min \left\{-b,-a_{i}-\sum_{j \in J_{i}} a_{j} \bar{u}_{j i}\right\} \quad \text { for } i \in I,
$$

then we have $0 \leq \lambda_{i} \leq-b$ for $i \in I \backslash \bar{I}$, and $\lambda_{i}<0$ for $i \in \bar{I}$. Also for $j \in J$, let $I_{j}:=\left\{i \in I: j \in J_{i}\right\}$; therefore, $I_{j}=\emptyset$ for $j \in J \backslash J^{-}$. The symmetric $n$-step mingling inequality is defined as

$$
\begin{aligned}
& \sum_{j \in J^{-}}\left(a_{j}+\mu_{\alpha,-b}^{n-1}(-b)\right)\left[x_{j}-\sum_{i \in I_{j}} \bar{u}_{j i} x_{i}\right]+\sum_{j \in J \backslash J^{-}}\left(a_{j}+\mu_{\alpha,-b}^{n-1}\left(-a_{j}\right)\right) x_{j} \\
& +\sum_{i \in I}\left(a_{i}+\sum_{j \in J_{i}} a_{j} \bar{u}_{j i}+\mu_{\alpha,-b}^{n-1}\left(\lambda_{j}\right)\right) x_{i}+s \geq b+\mu_{\alpha,-b}^{n-1}(-b)
\end{aligned}
$$

Theorem 3 For $n \geq 2$, the symmetric $n$-step mingling inequality (20) is valid for $K_{\geq}$ (with $b \leq 0$ ) for a sequence $\alpha$ satisfying $\alpha_{k}\left\lceil\frac{(-b)^{(k-1)}}{\alpha_{k}}\right\rceil \leq \alpha_{k-1}$ for $k=1, \ldots, n-1$, where $\alpha_{0}:=\max \left\{a_{j}: j \in J^{-}\right\}$. It is facet-defining for $\operatorname{conv}\left(K_{\geq}\right)$if the following conditions are satisfied:

(i) $(-b)^{(n-1)}>0$ and $\alpha_{k}=-a_{j_{k}}$ where $j_{k} \in J \backslash J^{-}$for $k=1, \ldots, n-1$;

(ii) $J^{-}=\left\{j \in J: a_{j} \leq \alpha_{1}\left\lfloor b / \alpha_{1}\right\rfloor\right\}$ and $\alpha_{k-1} \geq \alpha_{k}\left[\frac{(-b)^{(k-1)}}{\alpha_{k}}\right]$ for $k=2, \ldots$, $n-1$;

(iii) $u_{j_{1}} \geq\left\lceil\frac{-b}{\alpha_{1}}\right\rceil-\left\lceil\frac{\min \left\{\lambda_{i}: i \in \bar{I}\right\}}{\alpha_{1}}\right\rceil$ and $u_{j_{k}} \geq\left\lceil\frac{(-b)^{(k-1)}}{\alpha_{k}}\right\rceil$ for $k=2, \ldots, n-1$.

Proof The base inequality of $K_{\geq}$when $b \leq 0$ can be written as $\sum_{j \in J}-a_{j} x_{j}+$ $\sum_{i \in I}-a_{i} x_{i} \leq-b+s$ in the $K_{\leq}$form. The corresponding $K_{\geq}$form for this according to the Lemma 1 is $\sum_{j \in J}-\bar{a}_{j} x_{j}+\sum_{i \in I}-a_{i} x_{i}+s \geq-\bar{b}$. The $n$-step mingling inequality for this inequality is

$$
\begin{aligned}
& \sum_{j \in J^{-}} \mu_{\alpha,-b}^{n-1}(-b)\left[x_{j}-\sum_{i \in I_{j}} \bar{u}_{j i} x_{i}\right]+\sum_{j \in J \backslash J^{-}} \mu_{\alpha,-b}^{n-1}\left(-a_{j}\right) x_{j} \\
& +\sum_{i \in I} \mu_{\alpha,-b}^{n-1}\left(\lambda_{j}\right) x_{i}+s \geq \mu_{\alpha,-b}^{n-1}(-b) .
\end{aligned}
$$

Translating this inequality to the original $K_{\leq}$form using Lemma 1 gives the symmetric $n$-step mingling inequality. The facet-defining conditions are the direct result of Theorem 2 and Lemma 1.

As a special case, for $J^{-}=\emptyset$ the symmetric $n$-step mingling inequality (20) reduces

$$
\sum_{i \in N}\left(a_{i}+\mu_{\alpha,-b}^{n-1}\left(-a_{i}\right)\right) x_{i}+s \geq b+\mu_{\alpha,-b}^{n-1}(-b),
$$

which is equivalent to the negative $(n-1)$-step MIR inequality in [10]. 


\section{$6 \boldsymbol{n}$-step mingling cover and pack inequalities}

Inequalities described in the previous sections can be used in connection with complementing bounded variables to derive $n$-step mingling generalizations of cover and pack inequalities [3] for mixed-integer knapsack sets. Consider the mixed-integer knapsack set with bounded integer variables

$$
K_{\leq}^{u}:=\left\{(x, s) \in \mathbb{Z}_{+}^{N} \times \mathbb{R}_{+}: \sum_{i \in N} a_{i} x_{i} \leq b+s, x \leq u\right\},
$$

where $a_{i}>0$ for all $i \in N$. A subset $C$ of $N$ is a cover if $\beta:=\sum_{i \in C} a_{i} u_{i}-b>0$. After substitution $\bar{x}_{i}=u_{i}-x_{i}, i \in C$, the defining inequality of $K_{\leq}^{u}$ can be written as

$$
\sum_{i \in C} a_{i} \bar{x}_{i}+\sum_{j \in N \backslash C}-a_{j} x_{j}+s \geq \beta .
$$

Now for $\alpha=\left(\alpha_{1}, \ldots, \alpha_{n-1}\right)$ where $\alpha_{k-1} \geq \alpha_{k}\left\lceil\frac{b^{(k-1)}}{\alpha_{k}}\right\rceil$ for $k=2, \ldots, n-1$, by letting $I^{+} \subseteq\left\{i \in C: a_{i} \geq \alpha_{1}\left\lceil\beta / \alpha_{1}\right\rceil\right\}$, and $J=N \backslash C$, the $n$-step mingling inequality for the base inequality (23) can be written as

$$
\begin{aligned}
& \sum_{i \in I^{+}} \mu_{\alpha, \beta}^{n-1}(\beta)\left[\bar{x}_{i}-\sum_{j \in J_{i}} \bar{u}_{i j} x_{j}\right]+\sum_{i \in C \backslash I^{+}} \mu_{\alpha, \beta}^{n-1}\left(a_{i}\right) \bar{x}_{i} \\
& \quad+\sum_{j \in J} \mu_{\alpha, \beta}^{n-1}\left(\min \left\{\beta,-a_{j}+\sum_{i \in I_{j}} a_{i} \bar{u}_{i j}\right\}\right) x_{j}+s \geq \mu_{\alpha, \beta}^{n-1}(\beta) .
\end{aligned}
$$

We call inequality (24) the $n$-step mingling cover inequality. For $n=2$ inequality (24) reduces to the continuous integer cover inequality $[1,3]$.

Alternatively, a subset $P$ of $N$ is called a pack if $\theta:=b-\sum_{i \in P} a_{i} u_{i}>0$. After substitution $\bar{x}_{i}=u_{i}-x_{i}, i \in P$, the defining inequality of $K_{\leq}^{u}$ can be written as

$$
\sum_{i \in P} a_{i} \bar{x}_{i}+\sum_{j \in N \backslash P}-a_{j} x_{j}+s \geq-\theta .
$$

For $\alpha=\left(\alpha_{1}, \ldots, \alpha_{n-1}\right)$ where $\alpha_{k-1} \geq \alpha_{k}\left\lceil\frac{b^{(k-1)}}{\alpha_{k}}\right\rceil$ for $k=2, \ldots, n-1$, by letting $J^{-} \subseteq\left\{j \in N \backslash P: a_{j} \geq \alpha_{1}\left\lceil\theta / \alpha_{1}\right\rceil\right\}$, and $I=P$, the symmetric $n$-step mingling inequality for the base inequality above can be written as

$$
\begin{aligned}
& \sum_{j \in J^{-}}\left(-a_{j}+\mu_{\alpha, \theta}^{n-1}(\theta)\right)\left[x_{j}-\sum_{i \in I_{j}} \bar{u}_{j i} \bar{x}_{i}\right]+\sum_{j \in J \backslash J^{-}}\left(-a_{j}+\mu_{\alpha, \theta}^{n-1}\left(a_{j}\right)\right) x_{j} \\
& \sum_{i \in P}\left(a_{i}-\sum_{j \in J_{i}} a_{j} \bar{u}_{j i}+\mu_{\alpha, \theta}^{n-1}\left(\min \left\{\theta,-a_{i}+\sum_{j \in J_{i}} a_{j} \bar{u}_{j i}\right\}\right)\right) x_{i}+s \geq-\theta+\mu_{\alpha, \theta}^{n-1}(\theta) .
\end{aligned}
$$


We call inequality (25) the $n$-step mingling pack inequality. For $n=2$ inequality (25) reduces to the continuous integer pack inequality $[1,3]$. Facet-defining conditions for inequalities (24) and (25) can be easily derived using Theorem 2.

\section{Concluding remarks}

$n$-step mingling not only unifies two recent directions of research based on MIR ( $n$-step MIR and mingling) but also generates new valid inequalities and facets for the mixed-integer knapsack set utilizing the bounds on the variables and period MIR functions. The facet-defining property of $n$-step mingling inequalities for mixed integer knapsack sets suggests that these inequalities can be effective as cutting planes for solving MIPs. A particularly appealing feature of the $n$-step mingling inequalities is that, while their derivation is involved, they can be described in a simple compact form and be implemented using the combination of existing mingling and $n$-step MIR routines and constraint aggregation [13] routines to generate base inequalities.

Open Access This article is distributed under the terms of the Creative Commons Attribution Noncommercial License which permits any noncommercial use, distribution, and reproduction in any medium, provided the original author(s) and source are credited.

\section{References}

1. Atamtürk, A.: On the facets of the mixed-integer knapsack polyhedron. Math. Program. 98, 145-175 (2003)

2. Atamtürk, A.: Sequence independent lifting for mixed-integer programming. Oper. Res. 52, 487-490 (2004)

3. Atamtürk, A.: Cover and pack inequalities for (mixed) integer programming. Ann. Oper. Res. 139, 21-38 (2005)

4. Atamtürk, A., Günlük, O.: Mingling: mixed-integer rounding with bounds. Math. Program. 123, 315338 (2010)

5. Dash, S., Günlük, O.: Valid inequalities based on simple mixed-integer sets. Math. Program. 105, 29-53 (2006)

6. Gomory, R.E.: Some polyhedra related to combinatorial problems. Linear Algebra Appl. 2, 451-558 (1969)

7. Gomory, R.E., Johnson, E.L.: Some continuous functions related to corner polyhedra. Math. Program. 3, 23-85 (1972)

8. Gomory, R.E., Johnson, E.L.: Some continuous functions related to corner polyhedra, II. Math. Program. 3, 359-389 (1972)

9. Johnson, E.L.: On the group problem for mixed integer programming. Math. Program. Stud. 2, 137-179 (1974)

10. Kianfar, K., Fathi, Y.: Generalized mixed integer rounding inequalities: facets for infinite group polyhedra. Math. Program. 120, 313-346 (2009)

11. Kianfar, K., Fathi, Y.: Generating facets for finite master cyclic group polyhedra using $n$-step mixed integer rounding functions. Eur. J. Oper. Res. (2010, in press). doi:10.1016/j.ejor.2010.04.021

12. Marchand, H., Wolsey, L.A.: The 0-1 knapsack problem with a single continuous variable. Math. Program. 85, 15-33 (1999)

13. Marchand, H., Wolsey, L.A.: Aggregation and mixed integer rounding to solve MIPs. Oper. Res. 49, 363-371 (2001)

14. Nemhauser, G.L., Wolsey, L.A.: Integer and Combinatorial Optimization. Wiley, New York, USA (1988)

15. Nemhauser, G.L., Wolsey, L.A.: A recursive procedure to generate all cuts for 0-1 mixed integer programs. Math. Program. 46, 379-390 (1990)

16. Wolsey, L.A.: Integer Programming. Wiley, New York, USA (1998) 